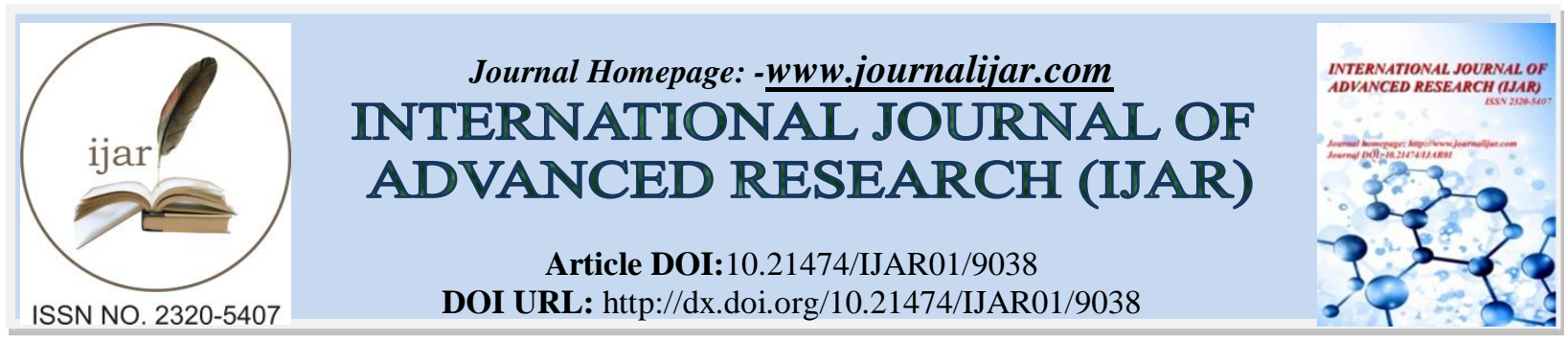

RESEARCH ARTICLE

\title{
UREJELEOMATINI KATIKA UWASILISHAJI WA MAUDHUI YA DHULUMA KATIKA RIWAYA YA MSICHANA WA MBALAMWEZI (K.W.WAMITILA).
}

1. Chuo Kikuu cha Egerton, Kenya.

\section{Janice M. Mutua ${ }^{1}$, Abdul Rahim Taib ${ }^{1}$ and Issa Mwamzandi² .}

2. Chuo Kikuu cha Kabianga, Kenya.

\section{Manuscript Info}

Manuscript History

Received: 10 March 2019

Final Accepted: 12 April 2019

Published: May 2019

\section{Key words:-}

Urejeleomatini, riwaya za kimajaribio, mbinu za kimajaribio.

\begin{abstract}
Waandishi wa riwaya ya Kiswahili ya kisasa wanawasilisha ujumbe kwa hadhira kwa kujikita katika mbinu za kiuandishi za kimajaribio. Urejeleomatini ni mojawapo wa vipengele muhimu vya mbinu hizo za kimajaribio. Riwaya zenyewe zimeitwa "riwaya za kimajaribio" au "riwaya za kisasa". Watalaamu wengine wamezibaini kama "riwaya za Karne ya Ishirini na Moja. Mwandishi Kyallo Wadi Wamitila ni mmojawapo wa waandishi wa kisasa anayetumia mtindo huu mpya wa kiuandishi katika kuzungumzia maswala mbalimbali yanayoikumba jamii. Nadharia ya usasaleo imeongoza utafiti huu katika uchanganuzi wa kipengele cha urejeleomatini. Aina mbali mbali za matini zilizorejelewa katika uwasilishaji wa maudhui ya dhuluma zilitambulishwa na kufafanuliwa. Riwaya ya Msichana wa Mbalamwezi ilichaguliwa kimaksudi kwa kuwa inawasilishwa kwa simulizi nyingi fupi fupi zinazorejelea matini tofauti tofauti. Mbinu ya uchanganuzi wa yaliyomo mkabala wa kimaelezo ilitumika kuchanganua deta iliyohusiana na swala zima la urejeleomatini. Deta ilichanganuliwa kwa kuangazia maudhui, mandhari na mbinu za kiuandishi za kimajaribio. Matokeo yaliwasilishwa kwa njia ya kimaelezo. Ilibainika kuwa ukosefu wa utaratibu katika uendeshaji wa mambo ndiyo taswira kamili ya jamii ya sasa. Matini zilizorejelewa zimeonyesha kuwa mwananchi amedhulumiwa na kupewa huduma duni na viongozi. Makala hii imehakiki na kutathmini jinsi mwandishi anavyotumia mbinu ya urejeleomatini kama kunga ya uwasilishaji wa maudhui ya dhuluma katika riwaya ya Msichana wa Mbalamwezi.
\end{abstract}

Copy Right, IJAR, 2019,. All rights reserved.

\section{Introduction:-}

1.1 Utangulizi

Makala hii imeshughulikia swala la urejeleomatini kama nyenzo ya kuwasilisha maudhui ya dhuluma katika riwaya ya Msichana wa Mbalamwezi (2011) ya Kyallo Wadi Wamitila. Lengo kuu ni kutambulisha aina mbalimbali za matini zilizorejelewa katika juhudi za kuwasilisha maudhui ya dhuluma. Mintarafu ya haya, makala imeonyesha jinsi riwaya ya kisasa ya Kiswahili inavyoisawiri taswira kamili ya jamii ya kisasa. Hali hii imejadiliwa katika misingi ya nadharia ya usasaleo.

Corresponding Author:-Janice M. Mutua.

Address:-Chuo Kikuu cha Egerton, Kenya. 


\subsection{Muhtasari wa Riwaya ya Msichana Wa Mbalamwezi}

Mhusika mkuu katika riwaya ya Msichana wa Mbalamwezi ni Yosi Kanga. Amekuwa na kazi nzuri na maisha mazuri. Siku moja hali ilimbadilikia punde tu aliposherehekea miaka thelathini na mitano tangu azaliwe. Yosi Kanga alitoka ofisini saa saba kwa mapumziko ya mchana. Alijilaza katika bustani kisha usingizi ukambeba. Aliota ndoto akiwa hatarini. Katika ndoto hiyo, banati fulani aliyemfahamu kama msichana wa mbalamwezi alimwokoa. Alielekea kujipa chakula cha mchana katika hoteli moja iliyokuwa karibu na makao makuu ya shirika la reli, Kenya Railways. Baada ya chamcha anaanguka ghafla na kuugua. Alipelekwa katika City Breeze Hospital na kulazwa mle.

Daktari alisema kwamba Yosi Kanga alikuwa na uvimbe kichwani na kwa sababu hiyo alikuwa na muda mfupi wa kuishi. Huduma mle hospitalini hazikuwa nzuri. Madaktari na baadhi ya wafanyikazi waliwadhulumu wagonjwa. Wengi wa wagonjwa hao walifariki kwa njia ambazo hazikueleweka. Baadaye daktari mwanamke alimtembelea. Mara alipomwona, Yosi alipatwa na hisia fulani. Alijaribu kukumbuka alikokuwa amemwona yule daktari. Daktari alimjulia hali na baada ya mazungumzo mafupi aliamua kumpasulia mbarika. Uchunguzi na "scan zote za ECG reports" zilionyesha kuwa Yosi alikuwa buheri wa afya. Daktari alimwagiza ajitayarishe kuondoka mle hospitalini. Yosi alifurahi sana kwa kuwa ile "hukumu ya kifo" aliyopewa mle hospitalini ilifutiliwa mbali. Kumbe huyu ndiye aliyekuwa mwokozi wake, Msichana wa Mbalamwezi.

\subsection{Usasaleo}

Riwaya ya Msichana wa Mbalamwezi imeandikwa katika misingi ya usasaleo. Elementi kuu ya usasaleo ni ukiukaji wa kaida za uandishi za kihalisia. Badala ya kuzingatia mfumo wa uhalisia mwandishi Kyallo Wadi Wamitila kama mwandishi wa kisasa ameandika riwaya hii kwa kuegemea uhalisia-mpya. Rettova (2016) amemtaja Wamitila kama mwandishi anayeegemea usasaleo kwa kuwa anaenzi matumizi ya mbinu za kimajaribio. Urejeleomatini ni kipengele muhimu katika mtazamo wa uandishi wa kisasaleo.

Wataalamu wamebaini kuwa ni vigumu kuweka mipaka kati ya usasa na usasaleo. Usasa ni kipindi katika historia ya fasihi ambacho kilifungamana sana na mabadiliko katika sanaa hasa kati ya miaka ya 1880-1930 barani Uropa. Wakati huo mwanadamu alidhihirisha uwezo na nguvu zake za kuumba, kuimarisha na kuunda mazingira yake kupitia mbinu mpya za kisayansi, teknolojia na majaribio (Best et al, 2001). Mabadiliko haya yalitokana na ukweli kwamba hapo awali fasihi ilitawaliwa na dini na siasa. Mabadiliko ya kisanaa yalihusisha tanzu za fasihi kama vile ushairi, tamthilia, riwaya, densi na filamu. Maudhui ya dhati, lugha ya dhati, uasilia, na utaratibu kabambe ndio mambo yaliyotiliwa maanani. Miji ya Uropa iliyoshuhudia mabadiliko haya ni pamoja na Paris, Vienna, Rome, London na Moscow. Mabadiliko haya yaliitwa Usasa na haya ndiyo yaliyokuwa kiini na msingi wa usasaleo. Abrams (1993) anashikilia kuwa Usasa hutumiwa kuelezea na kutambulisha sifa mpya za dhamira, tanzu, dhana na mitindo ya kifasihi inayohusishwa na miongo ya kwanza ya Karne ya Ishirini yaani baada ya Vita Vikuu vya Pili vya Ulimwengu. Abrams anaendelea kusisitiza kuwa sifa zinazorejelewa hutofautiana kutegemea wahakiki husika. Wamitila (2007) anasema,

"Licha ya tofauti hizo, wahakiki wengi wanakubaliana kuwa msingi wa sifa hizo ni kujitenga na misingi ya jadi ya sanaa na utamaduni wa Kimagharibi au ule uliokuwako".

Kwa mujibu wa haya, ni bayana kwamba usasa ulijitenga kabisa na taratibu za utunzi zilizofuatwa kabla ya Karne ya Ishirini. Hata ingawa suala la wakati wa kuzuka kwa usasa na usasaleo ni jambo la mjadala, ni wazi kwamba usasaleo uliibuka na kujengwa katika misingi ya usasa.

Usasaleo uliibuka na kuasisiwa katika Karne ya Ishirini. Ulihusishwa na Nietzchsche, Heidegger, Derrida, Lyotard, Baudrillard miongoni mwa wengine. Wataalamu wengine waliotoa michango yao kwa nadharia ya usasaleo ni pamoja na Jurgen Habermas (1987) ambaye anasisitiza kwamba ulimwengu wa sasa unajiegemeza zaidi kwenye maswala ya usasaleo ambapo shughuli zote zinazohusiana na binadamu wa sasa zinamulikwa kwa njia ya simulizi nyingi. Mfafanuzi wa hivi majuzi wa nadharia hii ni Fredric Jameson (1991). Makala hii imejikita katika maoni mbalimbali ya nadharia usasaleo kama ilivyoasisiwa na Jean - Francois Lyotard (1984), Jean Baudrillard (1994), Fredric Jameson (1991) na wengine.

Lyotard (1984) anashikilia nadharia tete kwamba ufahamu wa wanajamii umebadilishwa pakubwa na mabadiliko ya kipindi cha maendeleo ya viwanda. Hali hii imepelekea jamii kubadilisha tamaduni zake na kukumbatia tamaduni za kiusasaleo. Kipindi cha usasaleo kilivuruga mipaka iliowekwa na utamaduni wa jadi. Hali hii imejitokeza katika mambo mbalimbali yanayomilikiwa na jamii ikiwemo fasihi. Maisha na watu si wataratibu na kwa hivyo kazi za fasihi hazifai kuwa na utaratibu wowote. Usasaleo unafungamana sana na utamaduni pendwa ambapo watu hufanya 
mambo namna wanavyotaka wenyewe bila kufuata kanuni za kijadi. Mwandishi anajipa kibali cha kutumia kazi ya msanii mwengine kuundia kazi mpya. Mintarafu ya haya, makala hii imetambulisha aina za matini zilizorejelewa na mwandishi K.W.Wamitila katika riwaya ya Msichana wa Mbalamwezi kuwasilishia maudhui ya dhuluma. Isitoshe, imechanganua jinsi matini hizo zilivyotumika katika uwasilishaji wa maudhui ya dhuluma. Utafiti huu ulijikita katika kipengele cha urejeleomatini.

Carter (2006) anafafanua kwamba usasaleo hukiuka kaida na kanuni zote za utunzi. Kanuni anazorejelea Carter zaweza kuwa za kimuundo na za kilugha. Hivi ni kusema kwamba pamoja na fasihi pendwa, usasaleo huambatana na uigizaji pendwa. Sifa moja maarufu katika fasihi pendwa ni "mkondo wa ung'amuzi" ambao ni mbinu ya kisimulizi katika uandishi wa kinathari inayotumia maelezo marefu yanayoyamulika mawazo yanayompitikia mhusika akilini (Wamitila, 2006). Uigizaji huu hufanyika popote na haufuati utaratibu wowote. Aghalabu, lengo la mwandishi huwa ni kuburudisha jamii tu. Uigizaji pendwa hutumia lugha pendwa. Kwa kuwa lugha ni kifaa muhimu katika uandishi wa kazi za fasihi usasaleo unaangazia matumizi ya lugha kwa njia huru sana bila kuzingatia ufasaha na usanifu wake. Pia huhusu kuchanganya lugha mbalimbali kwa mfano Kiingereza na Kiswahili au lugha za kwanza na Kiswahili. Dhana hii iliuelekeza utafiti huu katika uchanganuzi wa maswala mahususi ya matumizi ya lugha pendwa hasa uchanganyaji na uhamishaji ndimi katika riwaya ya Msichana wa Mbalamwezi. Lugha iliyotumika hurejelea matini mbalimbali ambazo zinawasilisha maudhui ya dhuluma katika jamii. Msingi wa usasaleo ulitumika kuuongoza utafiti katika uchanganuzi wa urejeleomatini kama mojawapo wa kipengele muhimu cha riwaya za kimajaribio. Mtafiti amebaini jinsi kipengele hiki kilivyokosesha utaratibu katika riwaya teule.

\subsection{Urejeleomatini}

Urejeleomatini ni tafsiri ya neno la Kiingereza “intertexuality” lililotumika kwa mara ya kwanza na mwanafalsafa mfaransa, Julia Kristeva katika kitabu chake cha 'Dialogue and Novel' (1966). Kwa mujibu wa Kristeva, kila neno ni urejeleo wa maneno mengine. Hivyo, kuna msisitizo kwamba matini huhusiana na kutegemeana ili kuleta maana. Stam (2000) katika Anderson (2004:1) ananukuliwa,

"Matini yoyote ile iibukayo lazima iwe imelala pamoja na nyingine au na matini nyingine ambayo ile ya awali imelala nayo".

Usemi huu unadhihirisha kuwa kazi ya fasihi haitoki kwenye akili ya mtunzi pekee au katika ombwe tupu bali huwa ni mkusanyiko wa matini nyingine tofauti tofauti. (Anderson, 2004) anadai kuwa urejeleomatini huhusisha "yaliyosemwa tayari". Urejeleomatini ni kipengele muhimu cha usasaleo ambamo Wamitila anaandikia. Aidha, urejeleomatini ni mojawapo wa elementi kuu za uandishi wa kimajaribio. Urejeleomatini unaojitokeza aghalabu katika riwaya ya Msichana wa Mbalamwezi umeshamiri matumizi ya vihadithi vidogo vidogo, tanzu za fasihi simulizi, maoni ya wanafalsafa, ushairi/nyimbo na uchanganyaji wa lugha. Matini hizi zote zimetumika katika riwaya hii kuwasilisha maudhui ya dhuluma katika nyanja mbalimbali za jamii kama vile afya, elimu na mapenzi. Hali hizi zote huingiliana na kutegemeana katika ukuzaji wa ubunifu wa mwandishi.

Kwa mujibu wa Julia Kristeva (1980) matini huwa na uhusiano na matini nyingine. Makala hii imebaini kwamba katika uwasilishaji wa maudhui ya dhuluma katika riwaya ya Msichana wa Mbalamwezi matini mbalimbali zinahusiana, kutangamana na kutegemeana. Jilala (2016) anaeleza kuwa maana ya matini moja hutokana na matini nyingine. Katika fasihi maana imewakilishwa na maudhui (Mohammed, 1995). Urejeleomatini katika riwaya teule umejitokeza kiviwango kama vile fani, uhusika, dhamira na maudhui. Makala hii imetambulisha aina za matini zilizorejelewa katika uwasilishaji wa maudhui ya dhuluma.

Enani (2005) anadai kwamba urejeleomatini huathiri namna ya usomaji wa matini mpya. Riwaya ya Msichana wa Mbalamwezi imethibitisha kuwepo kwa mwingiliano wa kimtindo. Vipande vingi vilivyorejelewa katika riwaya hii ni vya mitindo tofauti na vimeathiri msuko na ploti. Hadhira inatiliwa huko na kutolewa kule. Msomaji anasoma kipande cha hadithi na kabla ya kukikamilisha kingine kinaanzishwa. Hali hii imepelekea kuleta uchangamano katika hadithi husika. Wamitila (2010) anadai kuwa urejeleo wa aina hii waweza kuzua kuwepo kwa kazi hasi au chanya.

Bakhtin (1981:271) wanadai kuwa yanayosemwa huwa ni zaidi ya matamko yenyewe. Miktadha mbalimbali huathiri kazi ya fasihi kwa njia tofauti. Miktadha kama vile ya kiuchumi, kijamii au kisiasa huathiri mahusiano fulani katika kazi za fasihi (Wamitila, 2010). Kwa mujibu wa haya, makala hii imechanganua na kuhakiki riwaya ya Msichana wa Mbalamwezi na kutambulisha aina za miktadha mbalimbali inayorejelewa, mahusiano yake na jinsi mahusiano hayo yanavyochangia katika kuwasilisha maudhui ya dhuluma. 


\subsection{Matinirejelewa na Uwasilishaji wa Maudhui ya Dhuluma katika Riwaya ya Msichana wa Mbalamwezi: Mtazamo wa usasaleo}

Nchi zinazoendelea huwadhulumu wananchi wake kwa njia tofauti tofauti. Njia hizo hujitokeza zaidi katika jinsi huduma zinavyotolewa. Kwa sababu mbali mbali ambazo wasanii huenda wakawa nazo, dhuluma hiyo huwasilishwa kibunifu kwa njia ya fasihi. Ili kueleza hali hii kikamilifu, mbinu na nyenzo za uandishi hutumiwa kwa ustadi na ufundi. Mwandishi K.W. Wamitila ametumia kipengele cha urejeleomatini ili kueleza uozo na dhuluma iliyomo katika jamii yake. Makala hii imeonyesha jinsi wananchi wa "kawaida" wanavyoteswa na maisha katika nchi yao kwa kukoseshwa huduma muhimu. Sehemu hii imeeleza kwa kina aina za matini zilizorejelewa na mwandishi katika mawasiliano yake na jamii kuhusu dhuluma.

\subsubsection{Matini za Kifalsafa}

Kulingana na Lyotard (1997), usasaleo unaegemea matumizi ya maoni ya kifalsafa katika utunzi. Shinikizo na changamoto za ndani hueleweka vyema zikihusishwa na falsafa au usomi fulani. Hata hivyo, matini za kifalsafa zinaweza kuwa changamano kwa wasomaji (Mwamzandi, 2013). Riwaya ya Msichana wa Mbalamwezi imehodhi bunilizi ndogo ndogo zinazorejelea maoni ya wanafalsafa. Falsafa ya utamaushi imempelekea mwandishi kukubali kuwa dhuluma anayopitia akiwa hospitalini ni hatua moja katika safari ya hatima yake ambayo ni kifo. Mazingira ya hospitalini alipolazwa Yosi Kanga ambaye ndiye mhusika mkuu katika riwaya hii yalikuwa mabaya. Mwandishi anasema kwamba vitanda vilikuwa vya zamani. Anaeleza hali hiyo kwa kusema,

“...vyuma vilivyolika na kuanza kuonyesha shumizi ya kutu hapa na pale, godoro dogo la usumba na kufunikwa shuka iliyochujuka rangi na kufanana na nguo iliyomwagiwa maji na kutoa mabakabaka”. (MWM. Uk. 25).

Mgonjwa anayerejelewa alikuwa ameshaambiwa na daktari kwamba alikuwa na muda mfupi wa kuishi. Kimsingi, hata mgonjwa akiwa katika hali mahututi anahitaji kupewa haki zake za kibinadamu. Hali iliyopo hospitalini inamtamausha zaidi. Hii ni kwa sababu inamdhulumu na kumnyima haki ya huduma bora hata ingawa alijua hatima yake ilikuwa kifo. Zaidi anadhulumiwa katika hisia zake na hadi anaafikia kujiliwaza kwa mawazo ya kitamaushi; “...nitapumzika nitakapokuwa mfu baada ya siku chache na kama watu wetu wasemavyo siku zilizohesabiwa ni chache - huisha kwa kufumba na kufumbua". (MWM. Uk.48).

Kwa mujibu wa Diegner (2005) falsafa ya kitamaushi inashikilia kuwa hatima ya binadamu ni kifo. Haijalishi iwapo ana kazi nzuri au la. Kando na kuwa na kazi nzuri iliyosimamia matibabu yake, suluhu ya pekee ya Yosi Kanga ilikuwa ni kifo.

\subsubsection{Matini za Kidini (Dini ya Kikristo, dini za kiasili)}

Yosi Kanga alikuwa na matatizo ya kiafya tangu jadi ambayo yalisababishwa na ajali za utotoni. Kila alipopatwa na shida alipelekwa hospitalini kwa toroli lenye gurudumu lililopiga kelele nyingi. Kila wakati watoto wa jirani waliposikia kelele ya gurudumu la toroli hiyo walijua Yosi anapelekwa hospitalini. Watoto hao walisimama karibu na ua wa seng'enge wa shamba lao la matunda na kumfanyia ishara za vicheko na dharau. Mwandishi anasema,

"Nilitamani ningelikuwa na uwezo wa Daudi wa Biblia. Niinuke kwenye ile toroli, nitafute tunguja mwitu au hata labda jiwe la tumbawe na kuwagotoa kwenye tosi za vichwa vyao na kuunyamazisha ugoliati wao" (MWM. Uk. 21).

Yosi aliwaona watoto wa majiranize kama Goliati wa Biblia aliyewadhulumu na kuwakejeli wana wa Isiraeli. Kwa mujibu wa dini ya Kikristo dhuluma ya Goliati kwa Waisraeli ilimalizwa na kijana mdogo, Daudi aliyelizungusha jiwe na kumwua. Yosi alitamani kuwa iwapo angekuwa na uwezo kama wa Daudi angewanyamazisha wale watoto waliomcheka na kumdharau. Aidha, vicheko vyao anavifananisha na Ugoliati.

Awali kidogo mhusika Konzi alimiliki hoteli moja ambamo alifanya kazi pamoja na mkewe. Mwandishi anaeleza kuwa Konzi alizoea kuwadhulumu wanawake lakini ugonjwa huo ulipona alipooa. Hata hivyo, aliendelea kumdhulumu mkewe kwa kuwatamani wanawake wengine waliokuja hotelini, machoni pa mkewe. Mke wa Konzi aliyeitwa Tabitha anaiona hali hii kama kutomtii Muumba. Anayerejelewa kama Muumba ni Mwenyezi Mungu. Kwa Tabitha dhuluma kama hii ni tendo la kutotii mojawapo wa amri kumi za Mungu inayoonya dhidi ya uzinifu kama ilivyorekodiwa katika Biblia (Kutoka 20). 
Tabitha alilipiza kisasi kwa mumewe kwa kumdhulumu zaidi. Mwandishi anasema kwamba Tabitha alipasua kicheko kikubwa akigeuka huku na kule kama mtu aliyetaka kuuomba ushirika wa wenzake. Mwishowe alimwuliza mumewe iwapo alimjua vizuri. Tabitha aliishia kwa kusema,

"Ikiwa wewe ni bafe, basi mimi ni bingwa wa kuitopoa sumu yake!” (MWM. Uk.51).

Matini inazorejelewa katika simulizi hii ni ya kishirikina. Mwandishi Wamitila anaeleza kuwa baada ya kioja hiki Bwana Konzi hakuweza kwenda haja kubwa wala kufanya haja ndogo. Hakuweza kufika hotelini tena,

"kuwaona hao wanawake wenye sura za nyanya wala kuwapakia siagi kwenye mikate yao" (MWM. Uk. 51).

Ni dhahiri kuwa mkewe alitumia nguvu za kishirikina kumroga mumewe katika juhudi za kumdhibiti kutokana na "jicho la nje". Isitoshe, Konzi alipopelekwa katika hospitali kuu ya Machakos madaktari walishindwa kumsaidia. Mwandishi anasema hata picha za uyoka (eksirei) hazikuonyesha tatizo lolote. Tumbo lake liliposikizwa kwa stethoskopu kilichosikika ni sauti iliyofanana na kuwika kwa jogoo. Alishauriwa na daktari kurudi nyumbani ambapo mkewe alicheka na kumzomea. Baadaye alimpiga kofi dogo shavuni na tatizo la Konzi likaisha. Imebainika kuwa Tabitha alitumia nguvu za kishirikina kumwadhibu yeyote aliyemvunjia heshima hasa mumwe Konzi. Jamii ikikosa mbinu mwafaka za kuipa haki inajitafutia mbinu zake. Katika muktadha huu, mbinu aliyotumia Tabitha inavunja utaratibu na maadili katika jamii. Kwa kawaida mke humtunza na kumheshimu mumewe kwa vyovyote vile.

\subsubsection{Matini za Kitaaluma}

Daktari alimwambia Mhusika Yosi Kanga kuwa tatizo la afya alilokuwa nalo lilikuwa la uvimbe uliokuwa kichwani. Alizidi kutaja kwamba hakuwa na siku nyingi za kuishi. Mwandishi ametumia maneno yafuatayo kueleza hali hii,

"Inavyoelekea ...mmm... ana tatizo la brain tumour... au hata intracranial hypertension...! Ikiwa diagnosis yangu ni sawa kwa sababu sijawahi kukosea katika miaka yote hii niliyofanya kazi ya udaktari, ana siku kama ishirini na nane za kuishi... in fact it might even be less!"

Matini za ujuzi wa kitaaluma zimetumiwa kumdhulumu Yosi kimawazo na kihisia. Uchunguzi haukufanywa vizuri kwa sababu baadaye inabainika kuwa Yosi alikuwa buheri wa afya. Daktari alijigamba kuwa msomi aliyesomea udaktari katika vyuo-tajika, “alumnus wa prestigious universities” (MWM. Uk. 31). Hivyo, alipozungumza kuhusu hali ya mgonjwa wake, Yosi alijua na kuamini ukweli kwamba siku zake duniani zilikuwa zimefikia ukingoni.

\subsubsection{Matini za kisiasa}

Hali ya siasa mara nyingi huwadhulumu wanajamii. Imebainika kuwa katika nchi nyingi zinazoendelea wanasiasa hutumia vijana kutekeleza matakwa yao. Mwandishi wa riwaya ya Msichana wa Mbalamwezi anasimulia kisa kinachowahusu wanajeshi. Anaeleza kuwa sura zao zilimkumbusha "mapando ya wanaume” waliotumiwa na matajiri kuwatishia wadeni wao (MWM. Uk. 106). Vilevile, mwandishi anasimulia kuhusu mhusika Moylee Gaga aliyenunua nyumba katika mtaa wa Golden Gate kwa mnada mwenye nyumba aliposhindwa kulipa rehani yake akiwa bado anaishi ndani. Mwenyeji alihitaji kuhama kutoka kwenye nyumba hiyo iliyopata bwana mwingine. Aliyeinunua hakujua amhamishe vipi hivyo Moylee Gaga alishauriwa atafute genge la vijana kama wafanyavyo wanasiasa, awape fedha wanunulie pombe kwenye Mtaa wa Madongokuinama kisha waende pale usiku na kuivurumisha nje mizigo ya mwenye nyumba bighairi ya vilio na kelele za jamaa yake (MWM. Uk. 106 - 107). Hapa, hata ingawa mwenyeji alishindwa kulipa rehani, kuhamishwa kwake kulifanywa kwa njia iliyomnyima iliyomdhulumu.

Matini nyingine ya kisiasa iliyotumika ni neno, “operesheni” (MWM. Uk. 107). Neno hili linadhihirisha jinsi jamii inavyoumia mikononi mwa wale wanaotarajiwa kuilinda. Moylee Gaga anazidi kushauriwa,

"hakikisha tu kuwa mkuu wa polisi wa eneo hilo anajua tu-nadhani unanielewa - kuwa una "operesheni" fulani tu; akijua tu basi huna la kuogopa kabisa. Kila jambo litakwenda sawasawa”.

Lugha ya kisiasa inabainika katika matumizi ya maneno na matendo ya wahusika mbali mbali waliotajwa katika muktadha huu. Njogu (1987) amebaini katika utafiti wake kwamba tabaka la chini (wanaoishi Madongokuinama) daima hupigana na ubwanyenye. Kinyume na utafiti huu, wanyonge wanaonekana kukubali hali yao. Isitoshe, wanakubali kutumiwa "vibaya" na mabwanyenye kwa kuhongwa na vitu vidogo vidogo kama pombe. Wenye nguvu wanatumia mamlaka yao vibaya kuwaumiza wengine walio katika tabaka la chini. Dhuluma iliyokithiri ni pale mwandishi anaposema kuwa, bora tu mkuu wa polisi ajue kuhusu “operesheni” fulani. Hiki ni kinyume cha 
matarajio ya jamii. Haya yanawiana na maoni ya Kamunde (1983) kwamba wanajamii wa tabaka la chini hudhulumiwa na wale wa tabaka la juu hata ingawa baadaye wao huzinduka na kuipigania haki yao.

\subsection{Hitimisho}

Makala hii imebaini kuwa maana, maadili na ukweli hupatikana bila kuegemea mbinu au upande mmoja kwa sababu chanzo chake haswa ni jamii. Dhuluma kwa jamii imewasilishwa kwa matumizi ya urejeleomatini ambacho ni kipengele cha uandishi wa kimajaribio. Matini mbalimbali zikiwemo zile za kifalsafa, kidini, kitaaluma na kisiasa zimetumiwa kuwasilisha maudhui ya dhuluma katika riwaya ya Msichana wa Mbalamwezi. Mwandishi ametumia vijisimulizi vidogo vidogo vyenye urejeleomatini mahsusi ili kuonyesha jinsi wanajamii wanavyonyimwa haki zao. Vilevile, imedhihirika kwamba jinsi maisha ya kisasa yanavyokosa utaratibu ndivyo fasihi ya kisasa ilivyo. Hata hivyo, ingawa hali ya ukosefu wa utaratibu katika utekelezaji wa shughuli mbalimbali waweza kuonekana kana kwamba haufai, unajitosheleza katika fasihi. Kutofaa huko ndiko maisha ya kisasa yaani taswira kamili ya jamii ya leo. Riwaya ya Kiswahili ya sasa inaafaa kwani ndiyo kioo halisi cha jamii ya leo.imebainika kuwa simulizi ndogo ndogo zinazorejelea matini tofauti tofauti zinafaa katika uwasilishaji wa maudhui badala ya matumizi ya hadithi moja ndefu iliyoandikwa katika msingi wa uhalisia. Mtendakazi yeyote yule ana uhuru wa kujaribu mbinu mpya za kutekeleza kazi yake bila kujibana na kujikita katika sheria za jadi. Hivyo basi, mtunzi wa kazi za fasihi ana uhuru wa kujaribu mbinu mpya za utunzi bila kubanwa na mbinu za kijadi ambazo zinajiegemeza katika msingi wa uhalisia. Hata hivyo, kuna hatari kwamba usomaji na uelewa wa riwaya ya kisasa unahitaji ufahamu wa kiada wa maswala mengi yanayohusiana na falsafa, taaluma mbalimbali, dini, siasa, lugha pendwa na mengine.

\section{Marejeleo:-}

1. Abrams, M.H. (1993). The Norton Anthology to English Literature II. New York na London: W.W. Norton.

2. Anderson, F.B., (2004). "Intertexuality and Memory in Yizo Yizo. Tasnifu ya Uzamifu, Chuo Kikuu cha Witwatersrand, Johannesburg.

3. Bakhtin, M. (1981). The Dialogic Imagination. Austin: University of Texas Press.

4. Baudrillard, J. (1994). Simulacra and Simulations. Ann Arbor: Michigan University Press.

5. Best, S. na Kellner D. (2001). The Postmodern Adventure. London, England, na New York: Routledge and Guilford Press.

6. Carter, D. (2006). Literary Theory. Harpenden, Herts, AL5 IX: Pocket Essentials.

7. Diegner, L. (2005). "Intertexuality in Contemporary Swahili Novel: Euphrase Kezilahabi's Nagona and William E. Mkufya's Ziraili na Zirani in Swahili Forum 12 (2005): 25-35.

8. Enani, M., (1996). Comparative Moments. Cairo: GEBO.

9. Habermas, J. (1987). The Philosophical Discourse of Modernity. Twelve Lectures, translated by Frederick Lawrence. Cambridge: The MIT Press.

10. Jilala, H. (2016). Misingi ya Fasihi Linganishi Nadharia, Mbinu na Matumizi. Dar es Salaam: Daud Publishing Company.

11. Kamunde, M.E. (1983). “Kilicho cha Wanyonge katika Riwaya za Said A. Mohammed”. Tasnifu ya Uzamili ya Chuo Kikuu cha Nairobi.

12. Kristeva, J. (1980). Word, Dialogue and Novel. Desire in Language: A Semiotic Approach to Literature and Art. Ed. Leon S. Roudies. New York: Columbia University Press. 64-91.

13. Kwambai, M.K. (2016). "Walenisi" and "Mafuta" Depiction of Class Segregation". Journal of Literature, Languages and Linguistics Vol 26.

14. Lyotard, J.F. (1984). The Postmodern Condition: A Report on Knowledge. Manchester: Manchester University Press.

15. Mohammed, S.A. (1995). Kunga za Nathari ya Kiswahili. Tamthilia, Riwaya na Hadithi Fupi. Nairobi: East African Publishers.

16. Mwamzandi I.Y. (2013). Riwaya Teule za Karne ya Ishirini na Moja na Udurusu wa Nadharia ya Fasihi. Swahili Forum 20 (2013): 48-66.

17. Njogu, K., (1987). "Maudhui ya Mapinduzi katika Riwaya za Visiwani Zanzibar". Tasnifu ya Uzamili ya Chuo Kikuu cha Nairobi.

18. Rettova, A. (2016). "Writing in the Swing? Neo-realism in Post- Experimental Swahili Fiction". Research in African Literatures, (47) 2, pp. 15-31

19. Stam, R., (2000). Film Theory: An Introduction. Oxford: Blackwell.

20. Wamitila, K.W. 2007). Narrative Structure and Metaphor: Towards a New Aesthetics of the Kiswahili Novel. KISWAHILI: 70: 67-752.

21. Wamitila K.W. 2011). Msichana wa Mbalamwezi. Nairobi: Vide-Muwa Publishers.

22. (2010). Kichocheo cha Fasihi: Simulizi na Andishi. Nairobi: English Press. 